\title{
AS CONTRIBUIÇÕES TEÓRICAS DE PINTRICH PARA PESQUISAS SOBRE A MOTIVAÇÃO DE ALUNOS NO ENSINO SUPERIOR
}

\author{
H. M. CARDOSO JÚNIOR ${ }^{1}$, T. W. A. SOUSA ${ }^{2}$, C. A. PEREIRA JÚNIOR ${ }^{3}$ \\ Instituto Federal de Educação, Ciência e Tecnologia de Mato Grosso ${ }^{1}$, Universidade Federal de Mato Grosso \\ do $\mathrm{Sul}^{2}$, Universidade Federal de Goiás ${ }^{3}$ \\ hjuniorgo@hotmail.com ${ }^{1}$
}

Artigo submetido 21/01/2018 e aceito 25/11/2019

DOI: $10.15628 /$ holos.2019.6851

\section{RESUMO}

Apesar do grande número de pesquisas na área de ensino sobre a motivação de alunos para a vida escolar, pouca atenção tem sido dada aos estudantes do nível superior, seja ela qual for a área do conhecimento. O estudo da temática "motivação no ensino superior" pode explicar a evasão, os altos índices de reprovação, o êxito nos cursos, além de outros fatos de relevante entendimento. Este trabalho tem como fim analisar teoricamente os fatores que influenciam a motivação dos alunos, focando a reflexão teórica em estudantes de graduação. Este estudo justifica-se para o embasamento de pesquisas que tratem da temática em questão, podendo ser um

aporte teórico para futuras pesquisas. Utilizou-se aqui como metodologia a pesquisa bibliográfica, caracterizando este estudo como exploratório. Destaca-se, para a motivação dos alunos é necessário que haja um planejamento para que as metas sejam atingidas. Uma vez que o professor é o proponente e também o mediador das intervenções propostas é necessário que ele se prepare para a nova fase que ele e turma viverão. A pesquisa aqui apresentada apresenta-se como aporte teórico para futuras pesquisas, utilizando-se os conceitos aqui desenvolvidos para traçar estratégias para análises diagnósticas e de intervenção pedagógica.

PALAVRAS-CHAVE: Êxito, Graduação, Intervenção, Pintrich.

\begin{abstract}
Despite the large number of researches in the area of teaching about the motivation of students to school life, little attention has been given to students of the upper level, whatever the area of knowledge. The thematic study "motivation in higher education" may explain the evasion, the high levels of disapproval, the success in the courses, as well as other relevant facts. This work is intended to analyze theoretically the factors that influence the motivation of students, focusing on the theoretical reflection on undergraduate students. This study is justified for the basement of research addressing the subject in
\end{abstract}

question, and may be a theoretical contribution to future research. It was used here as the bibliographical research methodology, characterizing this exploratory study. The highlight for the motivation of the students it is necessary that there is a plan for which the goals are achieved. Since the teacher is the proponent and also the mediator proposed interventions is necessary for him to prepare for the new phase that he and crew live. The research presented here is theoretical contribution to future research, using the concepts developed here to plot strategies for diagnostic and analysis of pedagogical intervention.

KEYWORDS: Success, Graduation, Intervention, Pintrich. 


\section{INTRODUÇÃO}

$\mathrm{Na}$ literatura do campo de ensino muito tem sido abordado sobre a questão da motivação de alunos em "aprender" nos mais diversos seguimentos do ensino. Porém ainda é frágil esse mesmo tipo de análise tendo como objeto de estudo os acadêmicos/estudantes do nível superior (Sassi \& Martinelli, 2010). E um provável motivo para essa observação é o fato das pesquisas serem feitas pelos próprios universitários, sendo comum o estudo do comportamento de alunos de outros níveis, principalmente o médio.

Um exemplo, dentre vários, é a pesquisa "Estudantes do Ensino Médio e a motivação para estudar Química" realizado por um grupo de pesquisa da UNIMONTES, Minas Gerais (Ribeiro, Fonseca \& Silva, 2004).

A motivação, independente de seus níveis, é um importante objeto de estudo no que se refere ao ensino e está ligada diretamente ao aprendizado e desenvolvimento dos alunos (Ruiz, 2005). Porém é perceptível que não há uma preocupação das entidades responsáveis em manter as condições mínimas para a permanência do acadêmico na universidade assim como o faz para os alunos nas escolas (Bzuneck, 2004).

Tendo como ponto de partida que o próprio acadêmico deve se direcionar e organizar para ser bem-sucedido nas atividades na Universidade, ou seja, aplicar a autorregulagem, este deve ser motivado para que seu aprendizado seja potencializado (Linnenbrink \& Pintrich, 2002). Deste modo, a autorregulação pode ser descrita como uma prática autodiretiva, em que os envolvidos potencializam as suas capacidades mentais em competências acadêmicas referentes às tarefas, podendo ser caracterizado como um processo ativo onde são estabelecidos objetivos que direcionaram a aprendizagem tendo como foco o conhecimento através de estratégias que privilegiam o monitoramento, regulação e controle do comportamento e, principalmente, a motivação (Joly et al, 2012).

Este artigo tem como objetivo realizar um estudo bibliográfico dos fatores envolvidos na "motivação" dos alunos de ensino superior durante a universidade, possuindo como base a teoria de Paul R. Pintrich (1953 - 2003). A pesquisa justifica-se em virtude de sua importância para as ações e planos de permanência e êxito nos diversos cursos do ensino superior, oferecendo um referencial teórico.

O trabalho está dividido em cinco seções, a contar com esta introdução. Nesse sentido, a segunda seção apresenta a metodologia utilizada nesta pesquisa; a terceira seção traz uma discussão sobre o conceito de "motivação"; a quarta seção presenta os fatores da motivação de alunos no ensino superior apresentados pela teoria de Paul R. Pintrich. Por fim, a quinta e última seção traz as considerações finais a respeito da discussão aqui apresentada. 


\section{METODOLOGIA}

Do ponto de vista dos objetivos aqui elencados, a pesquisa será desenvolvida de forma exploratória. Portanto, este trabalho envolve a pesquisa bibliográfica como seu principal passo metodológico. Nesse sentido, foi realizado um levantamento do tema nas principais obras nacionais e internacionais que tratam da temática, destacando, entretanto, os pressupostos da teoria de Paul R. Pintrich (1953 - 2003), pesquisador de Educação e Psicologia, como centrais para a definição dos fatores envolvidos no conceito de "motivação". Apensar de ser um conceito importante para os diversos níveis de ensino, neste trabalho destaca sua aplicação nos cursos de nível superior.

A maior dificuldade em se estudar a motivação e seus aspectos está no fato do número de variáveis que podem ser analisadas, tornando-o bastante complexo. Pintrich (2005) destaca que o entendimento da motivação de universitários é ainda mais importante que a de crianças e adolescentes, porque diferente destes os acadêmicos não são tão dependentes dos professores e devem buscar por si próprios o controle de seu comportamento, motivação e cognição. Isso porque a prática desta aprendizagem autorregulada vai ser importante não só no contexto acadêmico, mas em toda sua vida profissional.

\section{MOTIVAÇÃO ENQUANTO UM CONCEITO}

A palavra motivação tem origem do latim através da junção dos seguintes termos: verbo motum (mover) e o substantivo motivum (motivo). A junção destas duas palavras dá origem ao termo motivo (Bzuneck, 2009). Motivação pode ser entendida como tudo o que desperta, dirige e condiciona a conduta (Balancho \& Coelho, 1996), ou seja, pode ser considerada como um elemento fundamental para a utilização de recursos do indivíduo, com o intuito de se alcançar uma meta. São as metas que orientam a ação do indivíduo.

É muito comum que alguns conceitos sejam confundidos quando se trata de motivação, sendo bastante confundida também com rendimento e desempenho, termos bastantes utilizados neste contexto. Para melhor compreensão do trabalho, no Quadro 1 são explanados os conceitos de motivação, rendimento e desempenho, evidenciando suas diferenças.

Quadro 1: Síntese dos conceitos de motivação, rendimento e desempenho.

\begin{tabular}{|c|c|c|}
\hline Termo & Conceito & Autor \\
\hline Motivação & $\begin{array}{l}\text { Em abordagem operacional, motivação é o } \\
\text { conjunto de relações entre as operações de } \\
\text { estimulação ou privação e as modificações } \\
\text { observadas no comportamento que se processa } \\
\text { após as citadas operações. }\end{array}$ & Penna, 2001 \\
\hline
\end{tabular}




\begin{tabular}{|l|l|c|}
\hline Rendimento & $\begin{array}{l}\text { O rendimento é uma medida das capacidades do } \\
\text { aluno, que expressa o que este tem aprendido ao } \\
\text { longo do processo formativo. Também abarca a } \\
\text { capacidade do aluno em responder aos estímulos } \\
\text { educativos. Neste sentido, o rendimento escolar } \\
\text { prende-se com a aptidão. }\end{array}$ & Camargo, 1995 \\
\hline Desempenho & $\begin{array}{l}\text { O desempenho pode ser definido "como" as } \\
\text { pessoas utilizam suas competências e não se elas } \\
\text { "possuem" competências }\end{array}$ & $\begin{array}{c}\text { Verhine e Dantas, } \\
2010\end{array}$ \\
\hline
\end{tabular}

Fonte: Elaborado pelos autores.

O aumento na quantidade (e qualidade) das pesquisas que envolvem a motivação de alunos teve seu impulso nos anos 70, e a partir daí começou-se a desenvolver diversos modelos teóricos. Estes modelos focam em algum constructo ou dinâmica baseando-se em teorias filosóficas e/ou históricas e também pesquisas desenvolvidas pelos próprios pesquisadores, os quais serão citados adiante (Bzuneck, 2010).

A maioria destas teorias podem ser consideradas como teorias sócio-cognitivas, uma vez que levam em consideração as influências contextuais, culturais, demográficas no que tange a cognição na motivação de um indivíduo (Linnenbrink \& Pintrich, 2002). Com isso, prioriza-se o processo de regulação ativa, que parte do indivíduo, que envolve da sua motivação e que determina seu comportamento e pensamento (Schunk, 1992).

Tem-se que as principais teorias da atualidade sobre motivação no contexto escolar e acadêmico podem ser classificadas de acordo com em três questões que diferem bastante entre si, mas que estão inter-relacionados (Eccles \& Wigfield, 2002), sendo estas:

- teorias que focalizam expectativas (Posso fazer isso?);

- teorias que consideram as razões para o engajamento (Porque estou fazendo isto?); e

- teoria que integram os constructos expectativa e valor (Eu posso? Vale a pena?)

Quando o foco da motivação é o ambiente educacional este termo acaba tendo outros significados e conotações, tornando-se mais específico e preciso. Percebe-se que há uma forte ligação entre motivação, aprendizagem e desempenho neste campo (Pintrich, 2005).

Segundo Schunk (1992) a motivação tem interferência no quê, quando e como aprender, isso porque a motivação influencia a aprendizagem e o desempenho enquanto que as práticas e assimilações dos acadêmicos influenciam a motivação. Ou seja, é uma relação recíproca. A motivação é um dos assuntos que mais tem ganhado espaço no que se trata do bom desempenho de educandos do nível superior. Determinando, principalmente, a qualidade do envolvimento do aluno com o processo de ensino e aprendizagem.

Quando motivado o aluno busca novos conhecimentos, participa das atividades propostas com entusiasmo, aproveita oportunidades, mostra disposição para encarar novos 
desafios, tudo isso dando relevância a sua relação com o processo de aprendizagem. Sendo uma das principais variáveis relevantes do processo ensino/aprendizagem, a motivação busca explicar e melhorar o rendimento escolar, uma vez que este não pode ser explicado, único e exclusivamente por fatores como inteligência em si, contexto familiar e situação socioeconômica (Bzuneck, 2004).

A ideia de motivação deve ser entendida como um processo e não como um produto, sendo assim não pode ser analisada diretamente, mas pode ser estudada levando em consideração determinados comportamentos (Guimarães \& Boruchovitch, 2004).

Quando se analisa a motivação no campo da aprendizagem, é preciso levar em consideração as características específicas do contexto acadêmico. De modo geral, as propostas e atividades que se concretizam na universidade estão associadas a processos cognitivos, esses processos dizem respeito à capacidade de atenção, concentração, processamento de informações, raciocínios e resolução de problemas dos educandos.

Com isso, utilizar conceitos gerais sobre a motivação humana no âmbito escolar não é adequado sem levar em consideração as particularidades do ambiente. As teorias mais antigas traziam a motivação como uma pré-condição inerente a cada ser humano, porém estudos mais recentes mostram que os aspectos motivaciona is no campo educacional vão muito além desta pré-condição (Lens, Matos \& Vanteenkiste, 2008). É visível uma relação íntima entre motivação e aprendizagem de modo que a motivação pode interferir na aprendizagem e no desempenho, bem como a aprendizagem pode produzir um efeito na motivação (Schunk, 1992).

A motivação no ambiente acadêmico pode ser melhorada de duas formas: através de mudanças nos educandos e também através da mudança no seu ambiente de aprendizagem acadêmica. Aspectos da motivação podem esclarecer o fato de alguns acadêmicos gostarem e aproveitarem o seu tempo na Universidade, tendo comportamentos adequados, alcançando novas capacidades e desenvolvendo todo o seu potencial. A mesma motivação pode também explicar porque outros acadêmicos têm interesse raso nas atividades, na maioria das vezes sendo realizada por obrigação, ou de forma pouco responsável.

Alunos com falta de motivação pelas tarefas acadêmicas apresentam menor desempenho do que o que poderia conseguir com suas reais potencialidades (Bzuneck, 2004). É visível que se distraiam com facilidade, pouco participam das aulas, dedicam pouco (ou nenhum) tempo ao habito de estudar e o resultado disso tudo é o distanciamento do processo de aprendizagem. Ao terem baixo rendimento a chance de evasão do curso é grande fazendo com que suas oportunidades futuras sejam limitadas.

O professor, na sala de aula, deve ter como objetivo diário o desenvolvimento e a manutenção da motivação para aprender com as atividades acadêmicas (Bzuneck, 2004). O que é diferente da motivação para performance, que pode ser definida como um conhecimento ou habilidade que foi adquirida pelo aluno preocupando-se apenas com o desempenho final. É papel do professor mostrar aos alunos que devem considerar tais atividades significativas e merecedoras de envolvimento (Veiga, Resende \& Fonseca, 2000).

Trazer a motivação para o educando faz com que eles possam engajar-se nas 
atividades escolares, ainda que não considerem estas atividades prazerosas, encarando-as com seriedade, esforçando-se para aprender, desfrutando dos benefícios que o conhecimento pode trazer (Brophy, 1999). Cabe ressaltar que motivar é diferente de tentar fazer um controle do comportamento dos alunos, através da performance, priorizando a frequência obrigatória, as notas, tarefas, ignorando o processo e valorizando apenas o produto (Guimarães \& Boruchovitch, 2004).

\section{CONTRIBUIÇÕES TEÓRICAS DE PAUL R. PINTRICH}

A principal teoria que trata do conceito de motivação é a de Paul R. Pintrich (19532003), todavia pouco utilizada nos estudos no Brasil. Ele foi um pesquisador de Educação e Psicologia da Universidade de Michigan, sendo destaque no campo da Aprendizagem e da Instrução. Particularmente, sua pesquisa estava focada no desenvolvimento de motivação, mudança conceitual, pensamento epistemológico, aprendizagem autorregulada e no Ensino Superior. Em vida, publicou mais de 140 artigos e foi coautor ou coeditor de nove livros (LUQUE, 2004).

Pintrich é um dos autores, se não o principal, do Motivated Strategies for Learning Questionarie (MSLQ), um questionário que tem sido explorado com frequência em vários países e que quase não há relatos de sua utilização no Brasil. O princípio desta ferramenta está baseado na visão cognitivista de modo geral sobre a motivação (Luque, 2004). Para Pintrich, a motivação está atrelada a autorregulação, que o universitário pode ou não, bem ou mal, realizar em sua vida acadêmica.

Autorregulação é considerada como um processo consciente e voluntário de governo, pelo qual possibilita a gerência dos próprios comportamentos, pensamentos e sentimentos, ciclicamente voltados e adaptados para obtenção de metas pessoais e guiados por padrões gerais de conduta (Polydoro \& Azzi, 2008). Trata-se de um fenômeno multifacetado que opera por meio de processos cognitivos subsidiários, incluindo automonitoramento, julgamentos autoavaliativos e autorreações.

Este questionário tem como base a visão cognitivista sobre motivação e estratégias de aprendizagem considerando os educandos como processadores ativos da informação e que os sentimentos e cognições tem relevância no processo. No que tange a motivação, o modelo (Pintrich, 1991) incorpora quatro componentes principais, destacados a seguir:

a) Contexto Sócio-Cultural

De acordo com Pintrich (1991), o contexto sociocultural se refere a atitudes, crenças e comportamentos que os universitários carregam consigo para a faculdade, determinados por experiências anteriores. Tais aspectos estão relacionados com a integração e evasão dos acadêmicos e têm sido objeto de estudo de grande número de pesquisadores, quer seja em instituições públicas ou privadas, porém com foco no Ensino Superior. Essa realidade é vista 
não só no Brasil, mas no mundo, como exemplo destas pesquisas pode-se citar de Cote e Levine (1997), Pachane (1999), Polydoro e colaboradores (2001).

Nos Estados Unidos, uma pesquisa com foco nos aspectos socioculturais relacionados a integração a vida universitária, constatou que educandos com pais que não possuíam graduação e estudantes oriundos de minorias étnicas possuem maior dificuldade de habituação ao curso superior (Ratcliff, 1995).

Ainda se tratando dos aspectos socioculturais, estes têm o poder de interferir na forma como os acadêmicos encaram as atividades acadêmicas (Joly et al, 2012). Reglin e Adams (1990) comprovaram que nos Estados Unidos, alunos com descendência de populações asiáticas possuem tendência a investir maior tempo em propostas escolares do que em atividades de lazer, e fazem isso com o intuito de corresponderem às expectativas de sucesso de seus pais.

b) Fatores relacionados à sala de aula

Pintrich (1991) afirma que dentre os fatores relacionados à própria sala de aula podese citar, principalmente: os tipos de atividades propostas, a forma de lecionar do professor e as metodologias didáticas que adota. Estes fatores são importantes uma vez que a transição com êxito para a universidade está ligada com a qualidade de vida em sala de aula (Ratcliff, 1995).

Em contrapartida, mesmo reconhecendo sua relevância, deve ser enfatizado que grade parte das instituições de Ensino Superior ainda utilizam, em larga escala, métodos de ensinoaprendizagem tradicionais, especialmente os métodos mais diretivos, havendo a supervalorização da aula expositiva (Veiga, Resende \& Fonseca, 2000), onde os professores se restringem a repassar conteúdo, havendo pouca ou nenhuma preocupação em auxiliar os acadêmicos a aprender.

Se tratando do Ensino Superior brasileiro, o foco continua sendo no processo de ensino, ou seja, centrado no educador, enquanto deveria ter ênfase no processo de aprendizagem, com o aluno sendo o principal sujeito. O que deve ser feito é a complementaridade dos dois processos. Buscando-se evitar a metodologia tradicional de ensino, propostas têm sido oferecidas para a aprendizagem do acadêmico.

Travis (1996) explicita algumas dessas propostas: aprendizagem cooperativa, método de caso, teste de feedback e uso de vídeos. É então necessário que o corpo docente das instituições de Ensino Superior se aprimore para poder utilizar tais metodologias e ainda desenvolver outros métodos alternativos. Experiências inovadoras no Ensino Superior ainda não são suficientes para otimizar a motivação e são pouco difundidas entre os educadores das variadas disciplinas. Deixando-se de lado os métodos convencionais, a "metodologia da problematização", por exemplo, poderia ser utilizada buscando maior interesse dos acadêmicos. 
c) Fatores internos ao aluno

Também influencia a motivação dos universitários os fatores internos ao aluno, que de acordo Pintrich (1991) trata-se dos sentimentos, percepções, necessidades e metas de cada indivíduo e são relevantes na determinação da sua ação motivada e, consequentemente, seu desempenho acadêmico. É importante que, além do aluno exercer sua autonomia, o professor faça uso estratégias capazes de estimular os acadêmicos.

A motivação em aprender tem ligação com as crenças dos graduandos sobre a natureza da inteligência e do conhecimento. Alunos com crenças mais "sofisticadas" (que têm como verdade, por exemplo, que a inteligência é uma capacidade que pode ser desenvolvida, não sendo vista como uma entidade fixa) tendem a se esforçar mais e em diferentes estratégias aprendizagem (Ruiz, 2003).

O interesse que é despertado e a percepção do valor/utilidade de uma proposta acadêmica também interferem a motivação do graduando, por afetarem o nível de dedicação e persistência que a elas dedicam o aluno (Maciel, 2012). Alguns estudantes podem limitar seu envolvimento ou engajamento em determinada matéria e isso não significa, necessariamente, que são "desleixados", ou "desmotivados" (Ruiz, 2005). Estes alunos podem estar motivados a realizar apenas outras atividades diferentes das acadêmicas, tais como práticas esportivas, sociais, familiares e, ainda, de relacionamento com colegas (Sassi \& Martinelli, 2010).

Ainda é possível perceber acadêmicos que não possuem interesse particular e valorizam o curso que estão matriculados ou disciplinas que cursam da mesma forma (Dembo, 2000). A questão da motivação de universitários é buscar compreender as razões que os tornam desinteressados pelas atividades acadêmicas (Maciel, 2012). Pode-se citar como exemplos:

- a real preferência por realizar outras atividades, em vez de frequentar a faculdade;

- frequentar a faculdade como um fim em si mesmo e não para aprender;

- problemas pessoais (de saúde, psicológicos, financeiros, etc.);

- preguiça;

- falta de escolha vocacional;

- valores continuadamente imaturos.

d) Comportamento motivado em si

Este fator, o comportamento motivado em si, é caracterizado pelos comportamentos reais observáveis. $O$ acadêmico é responsável pela autorregulação da aprendizagem, processo que está relacionado à autoconscientização.

Pintrich destaca que no nível superior os estudantes já possuem capacidade de ter 
maior consciência de seu próprio comportamento, motivação e cognição, cabe ao acadêmico ter crenças motivacionais positivas e praticar estratégias autorreguladas de aprendizagem. Para alcançar tal patamar é necessário que as Universidades busquem práticas que enfoquem a pratica autorregulada, fazendo uso de metodologias eficientes (Pintrich, 1995).

Estudo aplicado a acadêmicos norte-americanos constatou que aqueles que se são intitulados como "alunos com baixas realizações" mostraram pouca ou nenhuma utilização de estratégias de autorregularem quando comparados àqueles com altas realizações.

Bembenutty e colaboradores (1998) pesquisaram como a ansiedade e a autoregulagem se relacionam com a motivação em acadêmicos e utilizaram como parâmetro de estudo o desempenho acadêmico. E o resultado da pesquisa foi que existem relações perceptíveis entre desempenho, ansiedade e auto regulação.

Estudantes que são considerados como ansiosos possuem algumas das seguintes características em comum, na maioria delas agem de forma imprópria, como exemplos (Dembo, 2000):

- Fazer tarefas pela metade;

- Conversar com colegas enquanto deveriam estar prestando atenção ou fazendo uma atividade;

- Fazer atividades depressa para poderem sair rapidamente da sala de aula ou adiarem sua realização.

Outro fator que pode influenciar o desempenho dos acadêmicos é a tentativa de proteger seu ego da possibilidade de fracasso, não realizando alguma atividade que julga difícil ou arriscada. Sabe-se, também, que o número de atividades que não são executadas devido esse receio se tornam mais frequente com o aumento da idade (Maciel, 2012).

Sweitzer (1999) afirma que para essa procrastinação universitária, inúmeros fatores podem afetar a motivação na vida acadêmica, citar algumas generalizações pode ser, no mínimo, imprudente. É, então, função do professor modelar e regular a aprendizagem e a motivação de educando, considerando suas características próprias, e também, enquanto grupo (Cavenaghi \& Bzuneck, 2009).

\section{CONSIDERAÇÕES FINAIS}

O estudo do campo da motivação nos mais diferentes cursos de graduação é de suma importância tendo em vista o objetivo os bons resultados. A noção de motivação permite a construção do conhecimento, a utilização de estratégias eficientes, bem como de métodos mais eficazes para o estudo no ensino superior. É visível que são necessários mais estudos que explorem a intervenção no campo da motivação, uma vez que os fatores contextuais têm forte 
influência na motivação dos acadêmicos. Logo é possível promover intervenções com o intuito de melhorar a qualidade do ato de motivar

A intervenção nas estratégias de aprendizagem é uma forma eficiente de modificar o desempenho de alunos em qualquer que seja o nível de ensino. Fazer a utilização de variadas técnicas para ensinar o aluno a aprender faz com que haja uma potencialização das experiências escolares e, também, traz a autoestima do acadêmico através de suas competências. Ao se sentirem mais seguros e capazes começam a despertar o senso de que possuem competência para estar sob seu controle de determinada situação.

A aplicação de intervenções pedagógicas, quando bem planejada, pode ser uma maneira eficiente de melhorar a motivação dos acadêmicos. Neste sentido, uma ferramenta bastante interessante é o debate, pois possibilita ao acadêmico aprimoramento da argumentação.

Neste contexto o maior desafio é destinado aos professores, que levando em conta as características dos estudantes universitários, devem promover a potencialização da motivação dos acadêmicos. Para isso é preciso evitar algumas características muito comuns no ambiente universitário: ênfase nas notas, o clima competitivo, a formação de grupos homogêneos por capacidade, as excessivas regras, a valorização da capacidade relativa, as poucas oportunidades de escolhas, as atividades desinteressantes e pouco desafiadoras, a relação mais impessoal entre professor e aluno e as rígidas formas de avaliação.

\section{REFERÊNCIAS BIBLIOGRÁFICAS}

Balancho, M.J.; Coelho, F. (1996). Motivar os alunos - criatividade na relação pedagógica: conceitos e práticas. Lisboa: Texto Editora.

Bembenutty, H.; Mckeachie, W. J.; Karabenick, S. A.; Lin, Y. (1998, maio). The relationship between test anxiety and selfregulation on student's motivation and learning. Reunião Anual da "American Psychological Society". Washington.

Brophy, J. (1994). Research on motivation in education: past, present and future. In: T.C. Urdan, M. Maehr, \& P.R. Pintrich, (Ed.), Advances in motivation and achievement p.1-44. Greenwich: Jai Press.

Bzuneck, J. A. (2009) A motivação do aluno: aspectos introdutórios. In E. Boruchovitch, J. A. Bzuneck (orgs.). A Motivação do Aluno: Contribuições da psicologia contemporânea. (4a ed., pp.9-36). Petrópolis, RJ: Vozes.

BZUNECK, J. A. (2010). Como motivar alunos: Sugestões práticas. In E. Boruchovitch \& J. A. Bzuneck (Orgs.), A motivação do aluno: Contribuições da psicologia contemporânea (3a ed., pp.58-77). Petrópolis, RJ: Vozes. 
CAMARGO, D. A. F. (1995). Avaliação do rendimento escolar: estudos e concepção (n. 8-9, pp.53-62). Ribeirão Preto, Paidéia.

Cote, J. E.; Levine, C. (1997). Student motivations, learning environments, and human aquisition: toward an integrated paradigm of student development. In Journal of College School Development. 38 (3), pp.229-43.

Dembo, M. H. (2000). Motivation and learning strategies for college success: a selfmanagement approach. Mahwah, New Jersey. Lawrence Erlbaum Associates Publishers.

Eccles, J. S; Wigfield, A. (2002). Motivational beliefs, values, and goals. In Annual Review of Psychology, v.53, pp.109-132.

Guimarães, S. E. R.; Boruchovitch, E. (2004). O Estilo Motivacional do Professor e a Motivação Intrínseca dos Estudantes: Uma Perspectiva da Teoria da Autodeterminação. Psicologia: Reflexão e Crítica, 17(2), p.143-150.

Joly, C. R. A.; Dias, A. S.; Almeida, L. S.; Franco, A. (2012). Autorregulação na Universidade. II Seminário Internacional "Contributos da Psicologia em Contextos Educativos". Braga: Universidade do Minho.

Lens, W.; Matos, L.; Vansteenkiste, M. (2008). Professores como fonte de motivação dos alunos: O quê e o porquê da aprendizagem do aluno. Educação, 31 (1), pp.17-20.

Linnenbrink, E.; Pintrich, P. (2002). Motivation as an enabler for academic success. School Psychology Review, 31(3), pp.313-327.

Luque, L. M. (2004). A tribute to Paul R. Pintrich's contributions to psychology and education. Eletronic Journal of Research in Education Psychology, 2 (1), pp.159-162.

Maciel, A. G. (2012). Motivação e intervenção em estratégias de aprendizagem para compreensão leitora. (Dissertação de Mestrado). Universidade Estadual de Londrina. Londrina, Brasil.

Mueller, R. J. (1992). Instrucional Psychology. Champaigh: Stipies.

Pachane, G. G. (1999). A experiência universitária a partir da percepção do aluno. Resumo de Comunicação Científica apresentado na XXIX Reunião Anual da Sociedade Brasileira de Psicologia. Ribeirão Preto.

Polydoro, S. A. J. et al. (2001). Desenvolvimento de uma escala de integração ao Ensino Superior. In Psico-USF, 6 (1), pp.11-18.

Penna, A. G. (2001). Introdução à motivação e emoção. Rio de Janeiro: Imago.

Pintrich, P., Smith, D., Garcia, T. \& Mckeachie, W. (1991). A manual for the use of the motivated strategies for learning questionnaire (MSLQ). National Center for Research to Improve. 
Pintrich, P. R. (1995). Understanding self-regulated learning. In: New directions for teaching and learning. v.63, pp.3-12.

Pintrich, P.R. (2005). The role of goal orientation in self-regulated learning. San Diego: Academic Press, pp.452-502.

Polydoro, S. A. J.; Azzi, R. (2008). Autorregulação: aspectos introdutórios. In A. Bandura, R. Azzi, S. A. J. Polydoro (orgs.). Teoria Social Cognitiva: conceitos básicos. Porto Alegre, Editora Artmed, pp.149-164.

Ratcliff, J. L. (1995). Realizing the potential: Improving postsecondary teaching, learning and assessment. The National Report of the National Center on Postsecondary Teaching, Learning and Assessment. University Park, the Center.

Reglin. G. L.; Adams, D. R. (1990). Why Asian American high school students have higher grade point averages and SAT scores than other high school students. The High School, p.143149.

Ribeiro, R. A.; Fonseca, F. S. A.; Silva, P. N. (2004, maio/junho). Estudantes do Ensino Médio e a motivação para estudar Química. XXVI Congreso Latinoamericano de Química. Salvador.

Ruiz, M. V. (2003). (a) Motivação na Universidade. In: Revista Estudos de Psicologia, PUC Campinas, 20 (2), pp. 15-24.

Sassi, A. G.; Martinelli, S. C. (2010). Relações entre Autoeficácia e Motivação Acadêmica. Revista PSICOLOGIA, CIÊNCIA e PROFISSÃO, 30 (4), pp.780-791.

Schunk, D. H. (1992). Self-efficacy and academic motivation. Education Psychologist, v.26, pp.207-231.

Sweitzer, N. G. (1999). "Fiddle-Dee-Dee, I'll think about it tomorrow": Overcoming academic procrastination in Higher Education. Tese de Mestrado. Califórnia, Universidade Biola.

Travis, J. E. (1996). Models for improving college teaching: a faculty resource.

Veiga, I.P.A.; Resende, M.L.G. E Fonseca, M. (2000). Aula universitária e inovação. In I.P.A. Veiga, \& M.E.L. M. Castanho (org.). Pedagogia universitária. Campinas: Papirus.

Verhine, R. E.; Dantas, M. V. A avaliação do desempenho de alunos de educação superior: uma análise a partir da experiência do ENADE. In J. A. C. LORDÊLO, M.V. DAZZANI (Org). Avaliação educacional: desatando e reatando nós [online]. Salvador: EDUFBA, pp.349, 2010. 